\title{
Identificación de servicios de los ecosistemas en comunidades asociadas a tres áreas marinas de pesca responsable del Golfo de Nicoya, Costa Rica
}

Identification of ecosystem services in communities associated with three Marine Areas of Responsible Fishing, Golfo de Nicoya, Costa Rica

Valeria Umaña Blanco

Escuela de Planificación y Promoción Social, Universidad Nacional, Costa Rica valeria.umana.blanco@una.ac.cr

Michael Steven Arroyo Zeledón

Escuela de Planificación y Promoción Social, Universidad Nacional, Costa Rica. michael.arroyo.zeledon@una.ac.cr

Recibido: 09/12/2021 • Aceptado: 09/06/2021

\section{Resumen}

Los servicios de los ecosistemas son fundamentales para la supervivencia del ser humano y su reconocimiento por parte de las comunidades constituye un primer paso hacia un aprovechamiento sostenible de estos. En este trabajo se analizó la identificación de servicios de los ecosistemas por parte de comunidades vinculadas a tres Áreas Marinas de Pesca Responsable (AMPR) del Golfo de Nicoya en Costa Rica, como contribución a la definición de alternativas productivas que permitan disminuir la presión de la actividad pesquera en la zona 
y mejorar el desarrollo socioeconómico. Para esto se aplicó una encuesta en las comunidades aledañas a las siguientes AMPR: Isla Venado, Isla Caballo y Paquera-Tambor. El $90 \%$ de las personas encuestadas dijo obtener servicios de los ecosistemas, lo cual representa una oportunidad para realizar un aprovechamiento racional de estos. Los servicios mayormente señalados por personas de estas comunidades reflejan un reconocimiento casi exclusivo de servicios de aprovisionamiento, particularmente el abastecimiento de productos de la pesca. Se considera valioso poner la mirada en emprendimientos productivos más sostenibles y en el fortalecimiento de la educación y conciencia sobre la significancia de los servicios de los ecosistemas, con el fin de garantizar el bienestar integral de las personas de las comunidades asociadas a AMPR.

Palabras clave: servicios de los ecosistemas, Áreas Marinas de Pesca Responsable, Golfo de Nicoya.

\section{Abstract}

Ecosystem services are fundamental for the survival of the human being and their recognition by the communities constitutes a first step towards their sustainable use. This paper analyzed the identification of ecosystem services by communities linked to three Marine Areas of Responsible Fishing in Golfo de Nicoya, Costa Rica, as a contribution to the definition of productive alternatives directed to reduce the pressure of the fishing activity in the area and improve the socioeconomic development. For this, a survey was applied in the surrounding communities of the following Marine Areas of Responsible Fishing: Isla Venado, Isla Caballo and PaqueraTambor. In general, $90 \%$ of the people involved in the survey claimed to get ecosystem services and this represents an opportunity to make rational use of such services. The services mostly mentioned by people from these communities reflect an almost exclusive recognition of provisioning services, particularly the supply of fishery products. It will be valuable to focus on new, more sustainable forms of production, as well as strengthening education and awareness of the significance of ecosystem services in order to guarantee the integral wellbeing of people in the communities associated with Marine Areas of Responsible Fishing. 
Key words: Ecosystem services, Marine Areas of Responsible Fishing, Golfo de Nicoya.

\section{Introducción}

El ser humano interactúa de manera dinámica con los ecosistemas y los servicios que estos brindan, ya sea los de soporte, de aprovisionamiento, regulación o culturales (Oropeza, Urciaga \& Ponce, 2015). Los servicios ecosistémicos contribuyen de manera directa e indirecta al bienestar humano, teniendo así un valor tanto económico como no monetario (Cavanagh et al., 2016). En todo el planeta, el medio marino y los servicios que este proporciona se han degradado significativamente producto del crecimiento poblacional y la alta demanda de bienes y servicios (Oropeza et al., 2015). Worm et al. (2006) plantean que la sobreexplotación, contaminación y destrucción de ecosistemas han causado cambios importantes en las zonas marino-costeras, como el colapso de especies y extinción de poblaciones enteras.

Costa Rica no escapa de la situación que enfrentan los ecosistemas marinos y sus servicios en el mundo. El Golfo de Nicoya, en la región Pacífico Central, es la zona pesquera de mayor importancia en el país, con 22 comunidades y más de 11000 pescadores (Chávez-Carrillo, 2018). Se caracteriza por la diversidad de peces e invertebrados, así como la existencia de manglares, arrecifes rocosos y estuarios (Salas et al., 2012 citado en García \& Heinen, 2016). Sin embargo, el excesivo esfuerzo de pesca incrementa de manera preocupante la presión sobre el medio marino en el Golfo, causando la reducción de tallas de los especímenes, al igual que el decrecimiento y emigración de poblaciones de especies comerciales (Sánchez-Jiménez, Morales-Ramírez, Samper-Villarreal \& Sánchez-Noguera, 2014).

Una de las alternativas que surgen en Costa Rica para asegurar el aprovechamiento de los recursos pesqueros a largo plazo ha sido la creación de las Áreas Marinas de Pesca Responsable (AMPR). De acuerdo con Ross, Lizano, Chacón y Castro (2018), estas empiezan a ser introducidas en la normativa nacional en el año 2008 como áreas con características biológicas, pesqueras o socioculturales importantes en las que se regula la actividad de pesca. Según estos mismos autores, el establecimiento de las AMPR plantea una modalidad de manejo participativo en conjunto con los pescadores, pues son estos usuarios y garantes de los recursos marinos. 
Según Sánchez-Jiménez et al. (2014), el uso de artes de pesca no selectivas y la poca intervención estatal en la administración de recursos marinos son algunos de los retos que tiene el país en relación con el manejo de recursos en las zonas marino-costeras. La regulación de la pesca desde el centro del país y el poco abordaje de problemas sociales subyacentes en estas zonas pueden provocar que el modelo de AMPR conduzca al desplazamiento de grupos vulnerables y la explotación de recursos en otros lugares (García \& Heinen, 2016). Las comunidades costeras con alguna modalidad de control para la pesca presentan una escasa participación en la toma de decisiones respecto a los recursos naturales (Sánchez-Jiménez et al., 2014), lo que limita la posibilidad de diversificación de las actividades dentro de estas áreas.

Para que las AMPR en el Golfo de Nicoya cumplan su propósito, una de las soluciones planteadas es que las comunidades desarrollen otras actividades productivas, distintas de la pesca. Es decir, que se beneficien de todos los servicios ecosistémicos existentes en la zona, no solamente de la provisión de peces. Los autores Santos-Martín et al. (2015) indican que el aprovechamiento de diferentes servicios de los ecosistemas puede mejorar las estrategias de gestión de recursos en las comunidades con AMPR. El implementar otras posibilidades de producción y reducir la presión sobre el recurso pesquero permite no solo una mayor protección del ambiente, sino el desarrollo de creatividad hacia nuevas actividades que mejoren las condiciones de las comunidades y la participación de segmentos menos favorecidos de la población (Pacheco-Urpí, Salas \& Sierra-Sierra, 2013).

El reconocimiento comunitario de otros servicios de los ecosistemas, como por ejemplo culturales, constituye un primer paso hacia un cambio de actitud para el aprovechamiento de estos servicios (Santos-Martín et al., 2015). En este trabajo se pretende analizar la identificación de servicios de los ecosistemas por parte de comunidades vinculadas a tres AMPR en el Golfo de Nicoya, como contribución a la definición de alternativas productivas que permitan disminuir la presión de la actividad pesquera en la zona y mejorar el desarrollo socioeconómico.

Esta área ha sido tradicionalmente dedicada a la pesca, siendo uno de los principales puntos de desembarque desde los años sesenta, con presencia de barcos camaroneros, sardineros, de captura de corvina y otras especies (Parada, Elizondo, Mejías, Espinoza \& Rodríguez, 2018). Según 
Chacón et al. (2007, citado en García \& Heinen, 2016) entre 1994 y el 2005 los desembarques en el Golfo representaron el $65 \%$ de la producción nacional, sobreexplotando así la mayoría de las especies comerciales. En las últimas décadas, el Golfo de Nicoya ha sido caracterizado por la reducción de poblaciones de ictiofauna, el aumento desmesurado de pescadores $\mathrm{y}$, en general, el deterioro de la calidad del ambiente (Pacheco-Urpí et al., 2013). El alto esfuerzo de pesca, junto al incremento en los efectos del cambio climático, ocasiona que la presión sobre los recursos marinos en esta área sea cada vez mayor (Ross et al., 2018).

\section{Materiales y métodos}

\section{Área de estudio}

El Golfo de Nicoya está ubicado al oeste de Costa Rica, en el Pacífico Central. Tiene una longitud de $80 \mathrm{~km}$ y un ancho de $50 \mathrm{~km}$, con un área de $1550 \mathrm{~km}^{2}$ (Pacheco-Urpí et al., 2013). Es un sitio idóneo para el establecimiento, reproducción, desove y crianza de peces, debido a sus características fisicoquímicas y las descargas de agua dulce provenientes del Río Tempisque; durante la época lluviosa, el aumento en la cantidad de nutrientes favorece el incremento en la cantidad de especies marinas en esta zona (Quesada, 2015).

Con el propósito de contribuir a la sostenibilidad del recurso marino y pesquero en la zona, se han establecido siete AMPR: Isla Caballo, Puerto Níspero, Palito Montero-Isla Chira, Isla Venado, Costa de Pájaros, Tárcoles y Paquera-Tambor (Chávez-Carrillo, 2018). El presente trabajo se llevó a cabo con habitantes de las cercanías de tres de estas AMPR: Isla Venado, Isla Caballo y Paquera-Tambor, entre las coordenadas geográficas $9^{\circ} 59^{\prime} 22^{\prime \prime} \mathrm{N}, 85^{\circ} 5^{\prime} 40^{\prime \prime} \mathrm{W}$ y $9^{\circ} 40^{\prime} 51^{\prime \prime} \mathrm{N}, 84^{\circ} 49^{\prime} 2^{\prime \prime} \mathrm{W}$, respectivamente (Figura 1). La primera tiene una extensión de $1.48 \mathrm{~km}^{2}$ y una población estimada de 359 habitantes. La segunda abarca un área de alrededor de $7 \mathrm{~km}^{2}$ y una población estimada mayor a 900 personas. Por último, Paquera-Tambor cuenta con una extensión de $200 \mathrm{~km}^{2}$ (Chávez-Carrillo, 2018). De acuerdo con el Índice de Desarrollo Social, las comunidades asociadas a AMPR tienen un bajo nivel de desarrollo relativo (MIDEPLAN, 2018). 


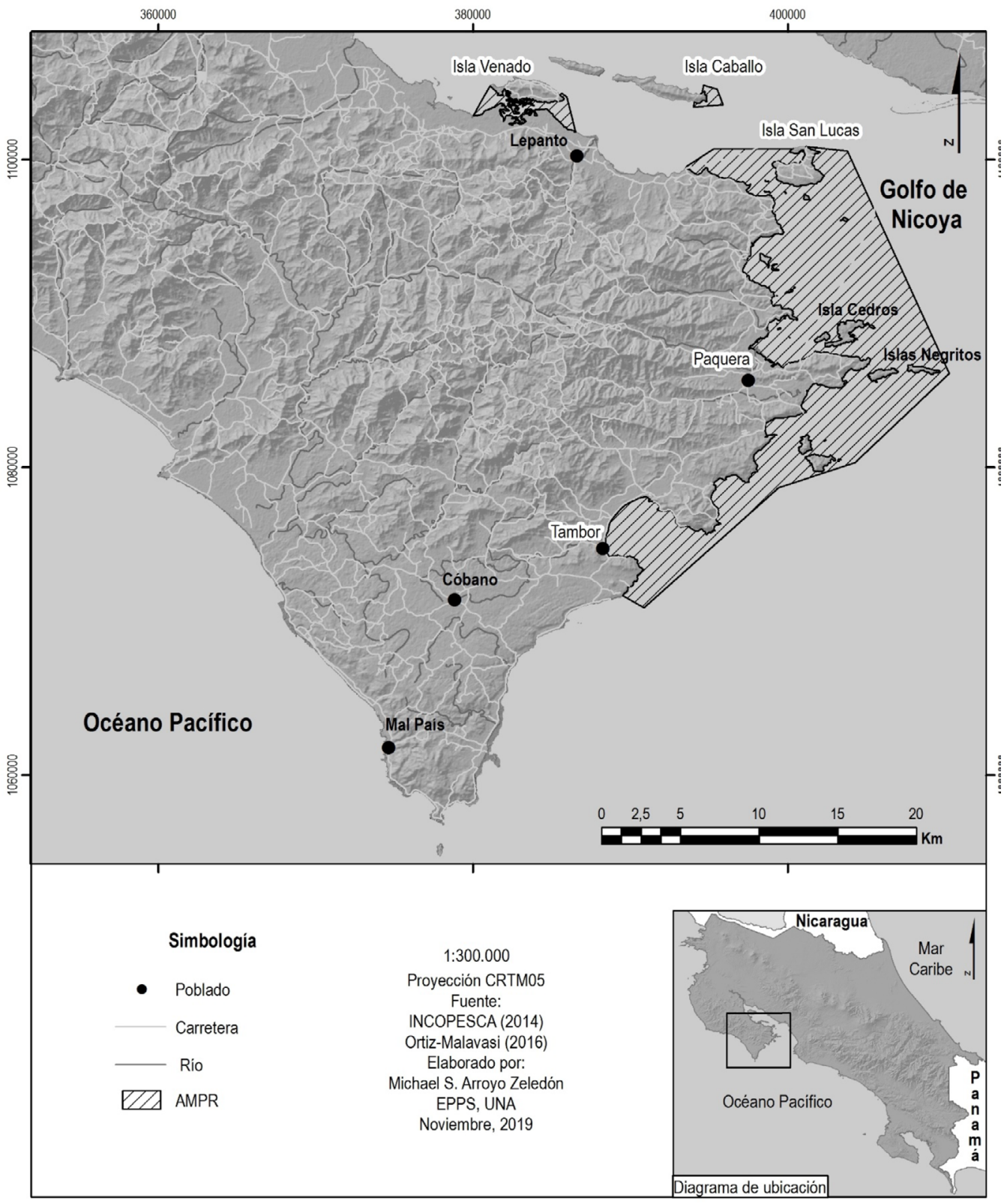

Figura 1. Ubicación de las Áreas Marinas de Pesca Responsable Isla Venado, Isla Caballo y Paquera-Tambor, Golfo de Nicoya, Costa Rica 
Para determinar qué servicios de los ecosistemas identificaba la población circundante a las tres AMPR se utilizó un cuestionario en el que también se consultaba por amenazas a tales servicios (ver anexo). La primera parte del cuestionario partía de información general sobre residencia y edad de los encuestados, así como si identificaban o no servicios ecosistémicos y amenazas a estos. Posteriormente, se les solicitaba que indicaran los servicios y amenazas específicos que reconocían.

El cuestionario se aplicó a una muestra no probabilística e intencionada compuesta por habitantes de las cercanías de las AMPR. Este tipo de muestra obedeció a que los encuestados debían ser mayores de 15 años y residentes de la zona, de manera que conocieran la realidad de esta última. Otros trabajos sobre servicios de los ecosistemas en un territorio han utilizado muestras de encuestados pequeñas (De Vreese., Leys, Fontaine \& Dendoncker, 2016).

La aplicación de cuestionarios ocurrió en marzo del año 2017. Estos fueron utilizados específicamente en las localidades señaladas en la Tabla 1. Como se aprecia, muchas más comunidades fueron consideradas para el AMPR Paquera-Tambor, debido a la extensión de esta. A cada encuestado se le brindó inicialmente una explicación, con ejemplos, del concepto "servicios de los ecosistemas". Con cada encuestado se tuvo interacción durante aproximadamente cinco minutos.

Tabla 1. Localidades asociadas a tres Áreas Marinas de Pesca Responsable (AMPR) del Golfo de Nicoya, Costa Rica, en las que se aplicaron cuestionarios para conocer los servicios ecosistémicos identificados por sus habitantes

\begin{tabular}{ll}
\hline AMPR & Localidad \\
\hline Isla Venado & Playa Florida \\
Isla Caballo & Playa Torres, Playa Rojas \\
Paquera-Tambor & $\begin{array}{l}\text { Punta Cuchillo, Isla Cedros, Barrio Paquira, Barrio las } \\
\text { Alinas, Muelle, Playa Blanca, La Leona, Punta del Río, } \\
\end{array}$ \\
& Tambor, Laberinto \\
\hline
\end{tabular}

Con las respuestas en los cuestionarios se construyó una base de datos como archivo .xls y a partir de esta se generó una distribución de frecuencia en cuanto a los servicios ecosistémicos más comúnmente mencionados. También se diseñaron gráficos para facilitar el análisis de los datos en 
cuanto a cantidad de servicios identificados y el número de amenazas a estos. Además, se dispuso en una tabla el nivel de importancia otorgado a los servicios más frecuentemente señalados.

\section{Resultados}

En total, se encuestó a 184 personas de comunidades asociadas a las AMPR Isla Venado, Isla Caballo y Paquera-Tambor. De estas personas, $42 \%$ tenía entre 30 y 50 años, $28 \%$ más de 50 años, $26 \%$ entre 20 y 30 años y $4 \%$ entre 15 y 20 años. Además, $82 \%$ de ellas tenían más de 10 años de residencia en sus localidades.

El $90 \%$ de las personas encuestadas dijo obtener servicios de los ecosistemas, mientras que el $10 \%$ restante indicó no hacerlo. La mayor parte de las personas identificó entre uno y dos servicios (Figura 2).

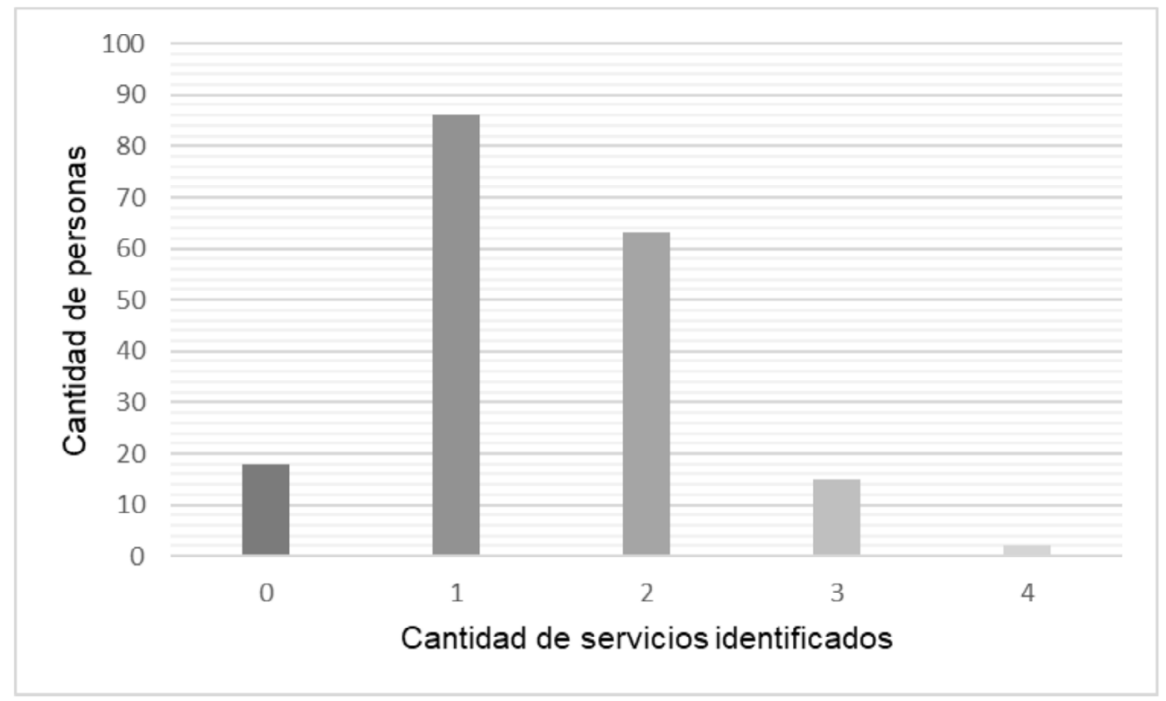

Figura 2. Cantidad de servicios ecosistémicos identificados por las personas encuestadas en localidades asociadas a tres Áreas Marinas de Pesca Responsable (Isla Venado, Isla Caballo y Paquera-Tambor) del Golfo de Nicoya, Costa Rica

\section{(ㄷ)(1)(3) (2)}


Un total de 12 servicios fueron identificados por las personas encuestadas. Los servicios mayormente mencionados fueron: abastecimiento de agua, abastecimiento de productos de la pesca y abastecimiento de alimentos en general. La mención de servicios en las categorías de regulación y culturales fue baja (Figura 3).

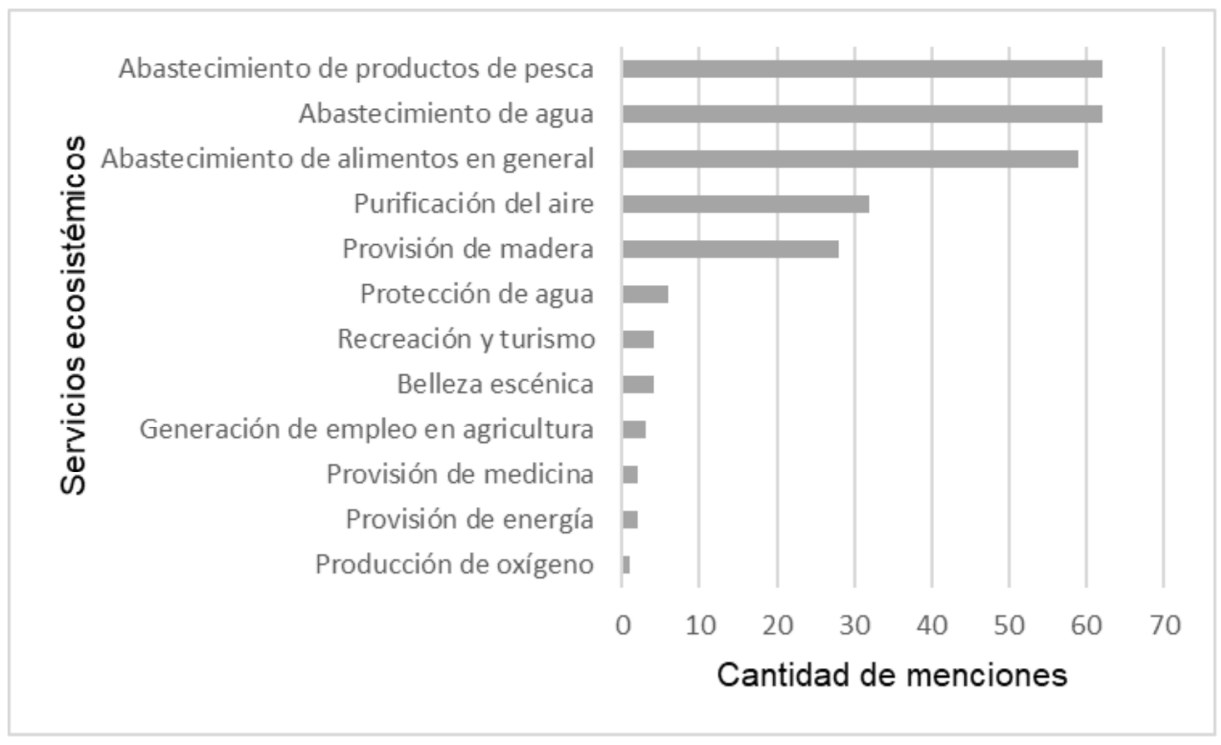

Figura 3. Servicios ecosistémicos identificados por las personas encuestadas en localidades asociadas a tres Áreas Marinas de Pesca Responsable (Isla Venado, Isla Caballo y Paquera-Tambor) en el Golfo de Nicoya, Costa Rica

Los tres servicios más mencionados por las personas encuestadas fueron, en su mayoría, catalogados por ellas como muy importantes. El abastecimiento de productos de pesca fue particularmente destacado. (Tabla 2).

\section{()(1)(2)}


Tabla 2. Nivel de importancia otorgado a los tres servicios ecosistémicos de mayor mención en localidades asociadas a tres Áreas Marinas de Pesca Responsable (Isla Venado, Isla Caballo y Paquera-Tambor) en el Golfo de Nicoya, Costa Rica.

\begin{tabular}{lccc}
\hline Servicio & \multicolumn{3}{c}{ Nivel de importancia } \\
\hline & $\begin{array}{c}\text { Muy } \\
\text { importante }\end{array}$ & Importante & Poco importante \\
\hline Abastecimiento de & 43 & 19 & 0 \\
productos de pesca & 32 & 15 & 0 \\
Abastecimiento de agua & 20 & 17 & 3 \\
Abastecimiento de & alimentos en general & 20 & \\
\hline
\end{tabular}

La mayor parte de las personas encuestadas, el 83\%, sí identificó amenazas para los servicios de los ecosistemas. De estas, la mayoría se refirió solamente a una amenaza (Figura 4).

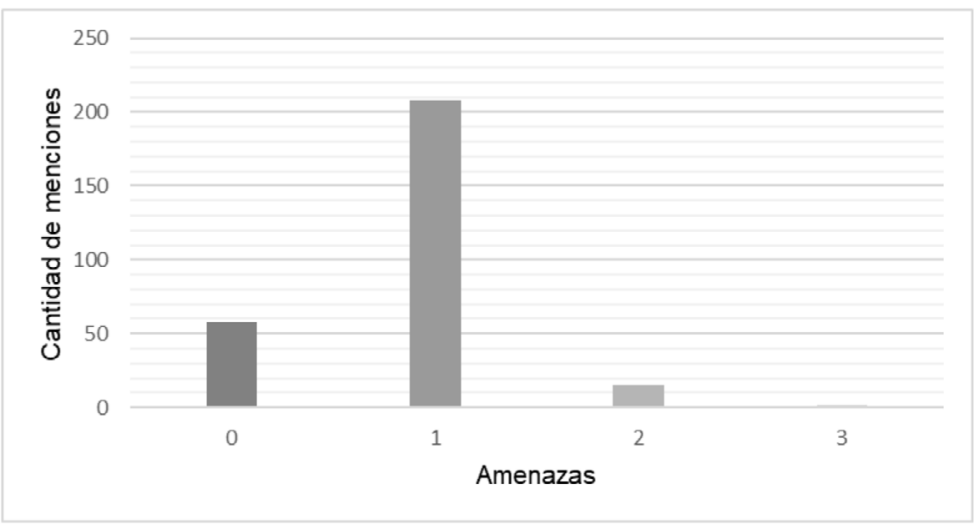

Figura 4. Cantidad de amenazas mencionadas para los servicios ecosistémicos identificados por las personas encuestadas en localidades asociadas a tres Áreas Marinas de Pesca Responsable (Isla Venado, Isla Caballo y Paquera-Tambor) en el Golfo de Nicoya, Costa Rica

Las amenazas más señaladas por las personas encuestadas para los tres servicios de mayor mención fueron: la contaminación, la pesca ilegal y artes inadecuadas, escasez y deforestación. Dichas personas asociaron 
estas amenazas con mayor frecuencia al servicio ecosistémico de abastecimiento de productos de pesca (Tabla 3 ).

Tabla 3. Amenazas más frecuentemente señaladas para los servicios ecosistémicos de mayor mención en localidades asociadas a tres Áreas Marinas de Pesca Responsable (Isla Venado, Isla Caballo y PaqueraTambor) en el Golfo de Nicoya, Costa Rica

\begin{tabular}{lccc}
\hline Amenaza & \multicolumn{3}{c}{ Servicio } \\
\cline { 2 - 4 } & $\begin{array}{c}\text { Abastecimiento de } \\
\text { productos de pesca }\end{array}$ & $\begin{array}{c}\text { Abastecimiento } \\
\text { de agua }\end{array}$ & $\begin{array}{c}\text { Abastecimiento } \\
\text { de alimentos en } \\
\text { general }\end{array}$ \\
\hline $\begin{array}{l}\text { Deforestación } \\
\text { Contaminación }\end{array}$ & 0 & 10 & 0 \\
Pesca ilegal y & 9 & 14 & 9 \\
artes inadecuadas & 21 & 7 & 5 \\
Escasez & 17 & 7 & 16 \\
Total & 47 & 38 & 30 \\
\hline
\end{tabular}

\section{Discusión}

El estudio de los servicios ecosistémicos se encuentra en un proceso de consolidación y discusión, con el fin de fundamentar una valoración de la naturaleza que genere la conciencia sobre su importancia y beneficios para la humanidad (Camacho-Valdez \& Ruiz-Luna, 2011). Pese a tomar relevancia en las últimas décadas, el concepto y la clasificación de estos servicios no ha logrado un acuerdo entre diferentes autores. Según De Groot, Alkemade, Braat, Hein y Willemen (2010) es difícil integrar este concepto en la planificación, gestión y toma de decisiones, sin antes resolver las discrepancias acerca de su definición y clasificación. Esto repercute en que la familiaridad con el concepto fuera de la comunidad científica sea baja; un hecho que fue evidente durante la aplicación de los cuestionarios en las comunidades asociadas a las AMPR, pues se presentó la necesidad de explicar con detalle el concepto.

El $90 \%$ de las personas encuestadas lograron identificar servicios de los ecosistemas, lo cual representa una oportunidad para realizar un aprovechamiento racional de estos. Tal como indican De Groot et al. (2010), las inversiones en conservación, restauración y uso sostenible de los 
ecosistemas pueden generar beneficios importantes a nivel social, económico y ecológico. Por lo tanto, no solo se pretende salvaguardar dichos servicios, sino también mejorar las condiciones socioeconómicas en las comunidades asociadas a las AMPR.

Autores como Mehvar, Filatova, Dastgheib, de Ruyter van Steveninck y Ranasinghe (2018), así como Lopes y Videira (2013), han señalado ejemplos de servicios ecosistémicos presentes en las zonas marino-costeras. Entre estos se encuentran la facilitación de actividades recreativas, mantenimiento de espacios con belleza escénica, provisión de plantas medicinales, fibra y combustible. Tales ejemplos constituyen también oportunidades para generar actividades económicas que beneficien a las comunidades cercanas y de allí parte de su gran importancia.

Los servicios mayormente señalados por personas de estas comunidades reflejan un reconocimiento casi exclusivo de servicios de aprovisionamiento, particularmente el abastecimiento de productos de la pesca. Estos servicios, al ser esenciales para la satisfacción de las necesidades humanas de nutrición, son impactados de manera negativa por el aumento de la demanda (Oropeza et al., 2015). En el caso del Golfo de Nicoya, algunos autores plantean que el deterioro ambiental en las zonas costeras se ha convertido en el común denominador de los últimos años, sin que se logre contrarrestar ni revertir este proceso de deterioro (Pacheco-Urpí et. al, 2013).

Por su parte, el servicio de abastecimiento de agua es otro de los más mencionados. Algunas de las problemáticas a las que se enfrenta la población cercana a AMPR en el Golfo de Nicoya respecto al recurso hídrico es la dudosa potabilidad y difícil acceso a este; pues es usualmente obtenido de pozos artesanales cuyo nivel desciende drásticamente en época seca (Artizar, Zabalza \& Carrillo, 2017). De igual forma, el tratamiento inadecuado de las aguas que drenan hacia el Golfo desde el río Tempisque, Barranca y Tárcoles, reduce la calidad del recurso en las comunidades aledañas (Valverde, 2013).

La poca mención de servicios en la categoría de regulación demuestra la falta de familiaridad que tiene la población con el concepto. Estos servicios intervienen en procesos ecológicos esenciales a través de ciclos bioquímicos, generando importantes beneficios para los seres humanos (Camacho-Valdez \& Ruiz-Luna, 2011). Dentro de estos servicios, según 
Corredor-Camargo, Fonseca-Carreño y Páez-Barón (2012), se incluye la regulación del clima y calidad del aire, el ciclo hidrológico y su calidad, así como la estabilidad en las temperaturas y precipitaciones; los cuales impactan directamente la salud y el bienestar de las personas a largo plazo. Estos mismos autores plantean que el mantener la integridad y resiliencia de los ecosistemas que proveen estos servicios es vital para superar la actual crisis ecológica.

Por otro lado, los servicios culturales son los menos reconocidos entre las personas encuestadas. Una explicación es que estos servicios son apreciados de una manera intuitiva y su relación con las necesidades humanas es indirecta y compleja (Daniel et al., 2012), de manera que su identificación, evaluación y gestión es un reto aún mayor. Además, los servicios culturales existentes en el Golfo de Nicoya no son tomados en cuenta dentro de los planes de desarrollo de la región, pues estos limitan el tema cultural a la creación de espacios artísticos, dejando de lado el arraigo existente hacia los ecosistemas que caracterizan a esta zona costera (Inder, 2015).

Las comunidades del Golfo de Nicoya se dedican, en mayor parte, a las actividades de pesca artesanal, seguida de la producción agropecuaria y turismo (Inder, 2015); siendo estas actividades impulsadas desde el Plan de Desarrollo Rural Sostenible y el Plan Estratégico Municipal de Puntarenas, con una serie de regulaciones para su adecuada ejecución. No obstante, se debe reflexionar acerca de la visión de desarrollo trazada para esta zona, pues estas actividades no han logrado reducir las disparidades que aquejan a sus habitantes. Esto último se ve reflejado en diversas estadísticas de pobreza y desarrollo social, las cuales posicionan a los distritos en los que se ubican estas comunidades con rezagos a nivel económico, educativo, de salud, entre otros (Mideplan, 2018). A pesar del potencial de zonas como esta, en general han sido abandonadas por diferentes sectores de la sociedad (Morales, Silva \& González, 2010).

Las zonas marino-costeras, en general, mantienen algunos de los hábitats más diversos y que proveen una amplia gama de servicios ecosistémicos de importancia para la sociedad (Caballero, 2015). Es entonces de suma relevancia tomar en consideración, dentro de la planificación para el 
desarrollo de estas comunidades, las particularidades de la zona y los servicios que pueden ser aprovechados. Se considera valioso poner la mirada en nuevas formas de producción más sostenibles y en el fortalecimiento de la educación y conciencia sobre la significancia de los servicios de los ecosistemas, con el fin de garantizar el bienestar integral de las personas de las comunidades asociadas a AMPR.

El pequeño tamaño de muestra involucrado en este estudio señala su carácter preliminar, pero al mismo tiempo lo convierte en un punto de partida para un trabajo de mayor alcance. En dicho trabajo podrá confirmarse la reducida familiaridad con el concepto de servicios ecosistémicos en las comunidades, así como que, en efecto, logran identificarse servicios distintos del asociado con la pesca. Sin embargo, de mayor importancia será que un estudio de mayor alcance analice la viabilidad de que las comunidades se beneficien económicamente de servicios ecosistémicos diferentes del vinculado con la actividad pesquera.

\section{Referencias}

Artizar, G. Zabalza, I. \& Carrillo, C. (2017). Pozos que hablan. La experiencia de gestión comunitaria del agua en Isla Caballo, vinculada al Programa de Desarrollo Integral de Comunidades Rurales Costeras del Golfo de Nicoya, Costa Rica. En Centroamérica: Agua, cultura y territorio. Actas del Primer Congreso Internacional. J. R. Rodríguez-Mateo (Ed.). Sevilla, España: Acer-VOS. Patrimonio Cultural Iberoamericano. Recuperado de: https://rio.upo.es/xmlui/bitstream/ handle/10433/5460/izurzu_carrillo.pdf?sequence=1\&isAllowed=

Caballero, N. (2015). Revisión del marco conceptual sobre Servicios Ecosistémicos. En Memoria de los foros técnicos sobre servicios ecosistémicos en Uruguay. N. Caballero \& M. T. Bolaños (Eds.). Montevideo, Uruguay: Instituto Interamericano de Cooperación para la Agricultura (IICA). Recuperado de: http://repiica.iica.int/DOCS/B3644E/B3644E.PDF\#page=9

Camacho-Valdez, V. \& Ruiz-Luna, A. (2011). Marco conceptual y clasificación de los servicios ecosistémicos. Revista Bio Ciencias 1(4), 3-15. doi: 10.15741/revbio.01.04.02 
Cavanagh, R., Broszeit, S., Pilling, G., Grant, S., Murphy, E. \& Austen, M. (2016). Valuing biodiversity and ecosystem services: a useful way to manage and conserve marine resources?. Proc Biol Sci, 283, (1844).. doi: 10.1098/rspb.2016.1635

Chávez-Carrillo, I. (2018). Diagnosing collective action in Small-Scale Fisheries for the establishment and management of Marine Areas of Responsible Fishing (AMPR) in the Gulf of Nicoya, Costa Rica. (Tesis de maestría). Turrialba, Costa Rica. Centro Agronómico Tropical de Investigación y Enseñanza (CATIE). Recuperado de: http:// repositorio.bibliotecaorton.catie.ac.cr/bitstream/handle/11554/8730/ Diagnosing_collective_action.pdf?sequence=1\&isAllowed=y

Corredor-Camargo, E., Fonseca-Carreño, J. \& Páez-Barón, E. (2012). Los servicios ecosistémicos de regulación: tendencias e impacto en el bienestar humano. Revista de Investigación Agraria y Ambiental 3(1), 77-84. Recuperado de: https://dialnet.unirioja.es/servlet/articulo?codigo $=4227147$

Daniel, T., Muharb, A., Arnberger, A., Aznar, O., Boyd, J., Chan, K., Costanza, R., Elmqvist, T., Flint, C., Gobster, P., Grêt-Regamey, A., Lave, R., Muhar, S., Penker, M., Ribe, R., Schauppenlehner, T., Sikor, T., Soloviy, I., Spierenburg, M., Taczanowska, K., Tam, J. \& Von der Dunk, A. (2012). Contributions of cultural services to the ecosystem services. PNAS, 109(23), 8812-8819. doi:10.1073/pnas.1114773109

De Groot, R.S., Alkemade, R., Braat, L., Hein, L. \& Willemen, L. (2010). Challenges in integrating the concept of ecosystem services and values in landscape planning, management and decision making. Ecological Complexity, 7(3), 260-272. doi: 10.1016/j.ecocom.2009.10.006

De Vreese, R., Leys, M., Fontaine, C.M. \& Dendoncker, N. (2016). Social mapping of perceived ecosystem services supply - The role of social landscape metrics and social hotspots for integrated ecosystem services assessment, landscape planning and management. Ecological Indicators, 66, 517-533. doi: 10.1016/j.ecolind.2016.01.048

García, A. \& Heinen, J. (2016). Identifying Drivers of Collective Action for the Co-management of Coastal Marine Fisheries in the Gulf of 
Nicoya, Costa Rica. Environmental Management, 57, 759-769. doi: 10.1007/s00267-015-0646-2

Instituto de Desarrollo Rural. (Inder 2015). Plan de Desarrollo Rural Territorial Territorio Peninsular Paquera-Cóbano-Lepanto-Chira 20152020. Costa Rica: Instituto de Desarrollo Rural (Inder). Recuperado de: https://www.inder.go.cr/territorio-peninsular/PDRT-Paquera-Cobano-Lepanto-Chira.pdf

Lopes, R. \& Videira, N. (2013). Valuing marine and coastal ecosystem services: An integrated participatory framework. Ocean \& Coastal Management, 84, 153-163. doi: 10.1016/j.ocecoaman.2013.08.001

Mehvar, S., Filatova, T., Dastgheib, A., de Ruyter van Steveninck, E. \& Ranasinghe, R. (2018). Quantifying Economic Value of Coastal Ecosystem Services: A Review. Journal of Marine Science and Engineering, 6(5), 1-18. doi: 10.3390/jmse6010005

Ministerio de Planificación Nacional y Política Económica. (MIDEPLAN 2018). Índice de desarrollo social 2017. Costa Rica: Ministerio de Planificación Nacional y Política Económica (MIDEPLAN). Recuperado de: https://www.mideplan.go.cr/indice-desarrollo-social

Ministerio de Planificación Nacional y Política Económica. (MIDEPLAN 2018). Índice de desarrollo social 2017. Región Pacífico Central. Costa Rica: Ministerio de Planificación Nacional y Política Económica (MIDEPLAN). Recuperado de: https://documentos.mideplan. go.cr/share/s/8AAfVqwWThy0GaY7J7yO8g

Morales, A., Silva, M. \& González, C. (2010). La gestión integrada de la zona costera en Costa Rica, experiencias y perspectivas. En Manejo costero integrado y política pública en lberoamérica: un diagnóstico. Necesidad de cambio. J.M. Barragán (Ed.). Cádiz, España: Red IBERMAR (CYTED). Recuperado de:https://www.researchgate.net/profile/Pedro-Alcolado-2/publication/265965017_El_Manejo_integrado_costero_en_Cuba_ un_camino_grandes_retos/links/544154fa0cf2a6a049a573ce/ El-Manejo-integrado-costero-en-Cuba-un-camino-grandes-retos. pdf\#page $=41$ 
Oropeza, M., Urciaga, J. \& Ponce, G. (2015). Importancia Económica y social de los servicios de los ecosistemas: Una revisión de la agenda de investigación. Revista Global de Negocios, 3(2) 103-113. Recuperado de: https://www.researchgate.net/profile/ Horacio_Santoyo/publication/274959924_Estrategias_de_Diferenciacion_en_Cuatro_Modelos_de_Negocio_de_Carne_Bovina/ links/552d5b690cf2e089a3ad747f.pdf\#page $=105$

Pacheco-Urpí, Salas, S. \& Sierra-Sierra, L. (2013). Modelo De Gestión Para La Sostenibilidad De Los Recursos Pesqueros Del Golfo De Nicoya, Costa Rica. Revista Geográfica De América Central, 1(50), 165-193. Recuperado de: https://www.revistas.una.ac.cr/index.php/ geografica/article/view/5395

Parada, M., Elizondo, S., Mejías, Y., Espinoza, J. \& Rodríguez, J. (2018). Análisis Socioeconómico de las Comunidades de Pesca a Pequeña Escala que Traslapan con la Flota de Arrastre en el Litoral Pacífico de Costa Rica. Fundación MarViva y Escuela de Economía de la Universidad Nacional de Costa Rica. Recuperado de: https://www.marviva.net/es/node/263

Quesada, V. (2015). Estudio de la comunidad de peces de importancia comercial y la abundancia planctónica en el Área marina de Pesca Responsable de Puerto Níspero, Guanacaste. Costa Rica. (Tesis de licenciatura). Heredia, Costa Rica. Universidad Nacional de Costa Rica.

Ross, E., Lizano, O., Chacón, D. \& Castro, M. (2018). Estudio de caso: Adaptación de las comunidades costeras vulnerables ante las amenazas inminentes del cambio climático en el área de Paquera, Puntarenas. Fundación MarViva. Recuperado de: https://fundecooperacion.org/wp-content/uploads/2020/10/Estudio-de-caso-MarViva.pdf

Sánchez-Jiménez, A., Morales-Ramírez, A., Samper-Villarreal, J. \& Sánchez-Noguera, C. (2014). Percepción comunitaria y procesos de Gestión Integrada de Zonas Costeras en el Pacífico Norte de Costa Rica. Rev. Biol. Trop. 62(4), 139-149. doi: 10.15517/RBT.V62I4.20040 
Santos-Martín, F., Montes, C., Alcorlo, P., García-Tiscar, S., González, B., Vidal-Abarca, M., Suárez-Alonso, M., Royo, L., Férriz, I., Barragán-Muñoz, J., Chica-Ruiz, A., López Santiago, C. \& Benayas, J. (2015). De la gestión de los recursos pesqueros a la gestión de los ecosistemas: La aproximación de los servicios de los ecosistemas aplicada a la gestión pesquera. Ambienta: La revista del Ministerio de Medio Ambiente, (111), 74-87. Recuperado de: https://dialnet.unirioja.es/servlet/articulo?codigo $=5457010$

Valverde, R. (2013). Disponibilidad, distribución, calidad y perspectivas del agua en Costa Rica. Revista de Ciencias Ambientales, 45(1), 5-12. doi: 10.15359/rca.45-1.1. Recuperado de: https://www.revistas.una. ac.cr/index.php/ambientales/article/view/7291

Worm, B., Barbier, E., Beaumont, N., Duffy, J.E., Folke, C., Halpern, B., Jackson, J., Lotze, H., Micheli, F., Palumbi, S., Sala, E., Selkoe, K., Stachowicz, J. \& Watson, R. (2006). Impacts of Biodiversity Loss on Ocean Ecosystem Services. Science, 314(5800), 787-790. doi: 10.1126/science. 1132294 